\title{
Screening of Natural Deep Eutectic Solvents for Green Synthesis of 2-methyl-3-substituted Quinazolinones and Microwave-Assisted Synthesis of 3-aryl Quinazolinones in Ethanol
}

\author{
Mario Komar, ${ }^{1}$ Maja Molnar, ${ }^{1, *}$ Anastazija Konjarević
}

\author{
1 Josip Juraj Strossmayer University of Osijek, Faculty of Food Technology, F. Kuhača 20, HR-31000 Osijek, Croatia \\ 2 Croatian Veterinary Institute-Branch Veterinary Institute Vinkovci, J. Kozarca 24, HR-32100 Vinkovci, Croatia \\ * Corresponding author's e-mail address: maja.molnar@ptfos.hr
}

RECEIVED: November 7, 2019 * REVISED: December 26, 2019 * ACCEPTED: December 28, 2019

\begin{abstract}
In this study, two fast and efficient protocols for green synthesis of 3-substituted quinazolinones were perfomed. A synthesis of 2-methyl-3-substituted quinazolinones was performed in natural deep eutectic solvents, while 3-aryl quinazolinones were obtained by using microwave assisted synthesis. Benzoxazinone, which was used as an intermediate in the synthesis of 2-methyl-3-substituted quinazolinones, was prepared conventionally from anthranilic acid and acetic anhydride. In order to find the most appropriate synthetic path, twenty natural deep eutectic solvents were applied as a solvent in these syntheses. Choline chloride:urea (1:2) was found to be the most efficient solvent and was further used in the synthesis of 2-methyl quinazolinone derivatives (2-12). 3-Aryl quinazolinones (13-17), on the other hand, were synthesized in one-pot microwave-assisted reaction of anthranilic acid, different amines and trimethyl orthoformate. All compounds were synthesized in good to excellent yields, characterized by LC-MS/MS spectrometry and ${ }^{1} \mathrm{H}$ - and ${ }^{13} \mathrm{C}-\mathrm{NMR}$ spectroscopy.
\end{abstract}

Keywords: natural deep eutectic solvents, microwave-assisted synthesis, quinazolinones, anthranilic acid, amine.

\section{INTRODUCTION}

Q UINAZOLINONES are widely used heterocyclic compounds of a great pharmaceutical interest. Due to their structural diversity, they possess various biological activities, including antihelmintic, ${ }^{[1,2]}$ antibacterial, ${ }^{[3-5]}$ anti-inflammatory, ${ }^{[6]}$ anticancer, ${ }^{[7,8]}$ antihyperlipidemic ${ }^{[9]}$ and antioxidant. ${ }^{[10]}$ Their biological activities have been recognized by pharmaceutical industry and today many commonly available drugs contain different quinazolinones as an active ingredient (Figure 1). Therefore, they are synthesized on a daily basis in many laboratories these days, and improving their synthesis in terms of making these processes "greener" is of a great importance.

Modern synthetic methods with green pathways are able to eliminate harmful solvents and they usually use mild conditions. Green synthetic methods are tending to comply with as many green chemistry principles as possible. ${ }^{[11]}$ In this manner, in the past few decades, a great attention is given to the design of the new solvents. Low-melting mixtures based on the naturally acceptable species, also called natural deep eutectic solvents (NADESs), are a novel generation of solvents in organic chemistry. They are often characterized as environmentally friendly and sustainable mixtures of nontoxic and cheap compounds, with low vapor pressure, non-flammability and biodegradability, where components form a eutectic mixture. Often, hydrogen bond acceptors (HBAs; quaternary ammonium salts) are combined with hydrogen bond donors (HBDs; ureas, polyols, dicarboxylic acids) in different ratios, to obtain DES with desirable physical and chemical characteristics. ${ }^{[12,13]}$

So far, we have successfully applied DESs in different organic synthetic routes, like in the Knoevenagel condensation of rhodanine derivatives with different aldehydes, ${ }^{[14]}$ synthesis of triazoles, ${ }^{[15]}$ Schiff bases ${ }^{[16]}$ and 2-mercaptoquinazolinones.[17] There are some extensive reviews

(c) BY This work is licensed under a Creative Commons Attribution 4.0 International License. 
describing the physico-chemical properties of the DESs, ${ }^{[18-22]}$ as well as their application in other synthetic routes. ${ }^{[23-25]}$ Apart from our report on the synthesis of 2-mercaptoquinazolinones in DESs, ${ }^{[17]}$ the synthesis of quinazolinone derivatives in DESs was also described by other authors. Lobo et al. (2012) synthesized 2,3-dihydroquinazolin-4(1H)one derivatives using choline chloride/malonic acid DES as a catalyst, ${ }^{[26]}$ while Zhang et al. (2012) used sugar/urea salt mixture as a solvent in the synthesis of quinazolinone derivatives. ${ }^{[27]}$ Ghosh et al. (2016) synthesized substituted quinazolinones and dihydroquinazolinones using $L-(+)-$ tartaric acid/dimethyl urea DES, ${ }^{[28]}$ and Arafa et al. (2019) synthesized bis-quinazolin-4-one derivatives in $L-(+)-$ tartaric acid/choline chloride DES. [29]

The aim of our work was to perform a synthesis of 3substituted quinazolinones using deep eutectic solvents. This method was applied for the synthesis of 2-methyl-3substituted quinazolinones. Since it was observed that the reaction of anthranilic acid and different orthoformates yielded no 3-substituted quinazolinones in DESs, a microwave-assisted synthesis was proposed for this reaction. Microwaves are often characterized as one of the green chemistry methods, due to the reduced reaction time, increase in the product yield and elimination of unwanted reaction pathways. The use of microwave-assisted synthesis is manifested in a huge increase of publications in the last decade, and a large number of quinazolinone derivatives have been synthesized, for the application in medicine and pharmacy. ${ }^{[30-33]}$ Therefore, in this paper we report two green protocols for the synthesis of 3-substituted and 2-methyl-3-substituted quinazolinones: a) deep eutectic solvents, and b) microwave induced synthesis, using anthranilic acid as a starting material.

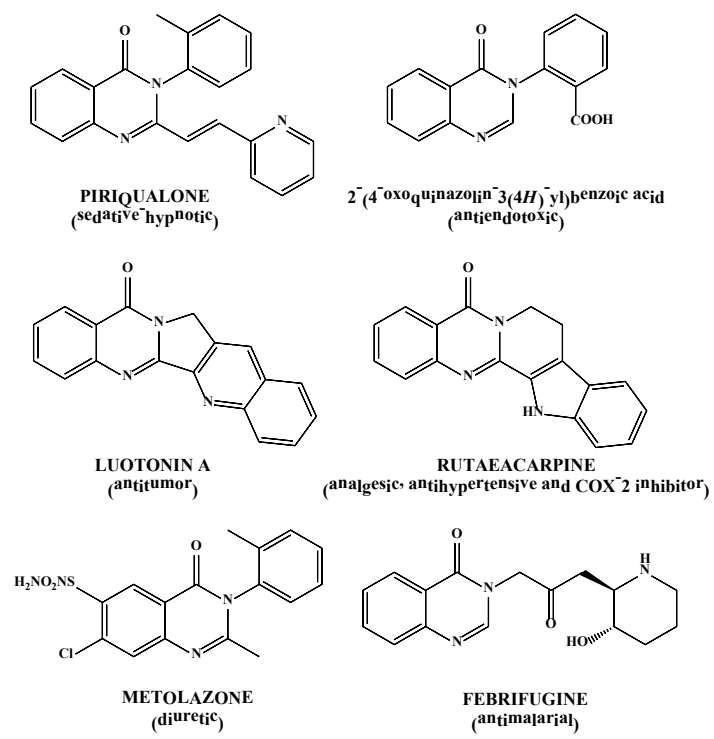

Figure 1. Several important quinazolinones in pharmacy. ${ }^{[34]}$

\section{EXPERIMENTAL}

All chemicals were obtained from commercial suppliers. All reactions were monitored by thin-layer chromatograhpy (TLC) using aluminium plates silica gel coated with flourescent indicator $\mathrm{F}_{254}$ (Kieselgel 60 ) in benzene : acetic acid : acetone $(8: 1: 1)$ as a solvent system. Microwaveassisted synthesis was performed in Milestone flexiWAVE (Milestone Srl, Sorisole (BG), Italy) microwave system, equipped with rotating carousel with 15 positions for PTFE high-pressure vessels. Melting points were determined using capillary Electrothermal IA9100 digital melting point apparatus. The mass spectra were recorded on the Agilent UPLC Infinity 1290 s QQQ 6460 Triple Quad LC/MS/MS, ESI, USA. All NMR spectra were recorded on a Bruker ultrashield Avance, working at 300 and $600 \mathrm{MHz}$ and chemical shifts are expressed as ppm in respect to the internal standard tetramethylsilane (TMS).

\section{Preparation of 2-Methyl-4H- benzo[d][1,3]oxazin-4-one}

A mixture of anthranilic acid $(0.1 \mathrm{~mol})$ and acetic anhydride $(0.2 \mathrm{~mol})$ was refluxed for 4 hours. The excess of acetic anhydride and acetic acid was evaporated under vacuum. The pale-yellow solid was washed with petroleum ether and used in the synthesis of 2-12.

\section{Preparation of NADES}

Choline chloride based DES were prepared as described by previous reports. ${ }^{[15,35]} \mathrm{A}$ mixture of choline chloride (7.5 g, $53.7 \mathrm{mmol}$ ) and different hydrogen bond donors (HBDs, Table 1) was stirred at $80^{\circ} \mathrm{C}$ until colorless liquid of NADES was obtained.

\section{General Procedure for the Synthesis of 3-(4-Chlorophenyl)-2-methylquinazolin- $4(3 H)$-one (1) and \\ 3-Aryl-2-methylquinazolin-4(3H)-one Derivatives (2-12)}

To the prepared DES containing molecular sieves $(1 \mathrm{~g}), 2$ methyl-4H-benzo[d][1,3]oxazin-4-one $(5 \mathrm{mmol})$ and 4-chloroaniline $(5 \mathrm{mmol}$ ) or other amine $(5 \mathrm{mmol} ; 2.5 \mathrm{mmol}$ of pyridine-2,6-dicarbohydrazide) were added. The reaction was stirred at $80^{\circ} \mathrm{C}$, monitored by TLC, and after the completion of the reaction, water was added. Molecular sieves were removed and crude product was collected by filtration. The desired product was recrystallized from ethanol.

\section{General Procedure for the Synthesis of 3-Substituted Quinazolin-4(3H)-one Derivatives (13-17)}

Anthranilic acid $(5 \mathrm{mmol})$, trimethyl orthoformate $(6 \mathrm{mmol})$ and amine $(6 \mathrm{mmol})$ were mixed together and reaction was 
carried out by a microwave irradiation for $30 \mathrm{~min}$ at $120^{\circ} \mathrm{C}$. After the completion of the reaction, the mixture was poured over crushed ice and water. The crude product was collected by filltration and washed with cold water.

\section{3-(4-CHLOROPHENYL)-2-METHYLQUINAZOLIN- 4(3H)-ONE (1) ${ }^{[34]}$}

Yield: $89 \% ; \mathrm{Mp}=159-161^{\circ} \mathrm{C}$ (lit. $\left.159-160^{\circ} \mathrm{C}\right) ; \mathrm{Rf}=0.70$; ${ }^{1} \mathrm{H}-\mathrm{NMR}\left(300 \mathrm{MHz}, \mathrm{ppm}\right.$, DMSO- $\left.d_{6}\right): 2.14\left(\mathrm{~s}, 3 \mathrm{H}, \mathrm{CH}_{3}\right), 7.51-$ 7.54 (m, 3H, arom.), 7.63-7.68 (m, 3H, arom.), 7.85 (m, 1H, arom.), 8.08 (dd, $J=7.91,1.5 \mathrm{~Hz}, 1 \mathrm{H}$, arom); ${ }^{13} \mathrm{C}-\mathrm{NMR}$ (DMSO- $d_{6}$ ) $\delta$ (ppm): 161.92, 154.6, 147.8, 137.2, 135.1, 134.1, 130.9, 130.1, 127.1, 126.9, 126.8, 120.9, 24.5; MS: $\mathrm{m} / \mathrm{z}: 271.20(\mathrm{M}+)(270.71)$.

\section{3-(4-METHOXYPHENYL)-2-METHYLQUINAZOLIN- 4(3H)-ONE (2) ${ }^{[34]}$}

Yield: $71 \%$; $\mathrm{Mp}=170-173{ }^{\circ} \mathrm{C}$ (lit. $\left.167-170{ }^{\circ} \mathrm{C}\right) \mathrm{Rf}=0.62 ;{ }^{1} \mathrm{H}-$ NMR (300 MHz, ppm, DMSO- $\left.d_{6}\right): 2.15\left(\mathrm{~s}, 3 \mathrm{H}, \mathrm{CH}_{3}\right), 3.85$ (s, $\left.3 \mathrm{H}, \mathrm{OCH}_{3}\right), 7.01-7.12(\mathrm{~m}, 2 \mathrm{H}$, arom.), 7.34-7.37 (m, $2 \mathrm{H}$, arom.), 7.51 (m, 1H, arom.), 7.66 (m, 1H, arom.), 7.84 (m, $1 \mathrm{H}$, arom.), 8.09 (dd, $1 \mathrm{H}, J=8.10,1.32$, arom); ${ }^{13} \mathrm{C}-\mathrm{NMR}$ $\left(\mathrm{DMSO}_{6} \mathrm{~d}_{6}\right) \delta(\mathrm{ppm}):$ 162.0, 159.7, 155.5, 147.9, 134.9, 130.8, 129.9, 127.1, 126.8, 115.2, 55.9, 24.5; MS: $m$ / z: $267.10(\mathrm{M}+)(266.29)$.

\section{3-(4-BROMOPHENYL)-2-METHYLQUINAZOLIN- 4(3H)-ONE (3) $)^{[34]}$}

Yield: $53 \%$; $\mathrm{Mp}=170-173^{\circ} \mathrm{C}$ (lit. $\left.168-169{ }^{\circ} \mathrm{C}\right) \mathrm{Rf}=0.69 ;{ }^{1} \mathrm{H}$ NMR (300 MHz, ppm, DMSO- $\left.d_{6}\right): 2.14\left(\mathrm{~s}, 3 \mathrm{H}, \mathrm{CH}_{3}\right), 7.51-$ 7.54 (m, 3H, arom.), 7.63-7.68 (m, 3H, arom.), 7.85 (m, 1H, arom.), 8.08 (dd, $J=7.91,1.5 \mathrm{~Hz}, 1 \mathrm{H}$, arom); ${ }^{13} \mathrm{C}-\mathrm{NMR}$ $\left(\mathrm{DMSO}-d_{6}\right) \delta$ (ppm): 161.92, 154.6, 147.8, 137.2, 135.1, 134.1, 130.9, 130.1, 127.1, 126.9, 126.8, 120.9, 24.5; MS: $m / z: 314.80(\mathrm{M}-)$ (315.16).

\section{3-(2-BROMO-4-METHYLPHENYL)-2-METHYLQUINAZOLIN- 4(3H)-ONE (4)}

Yield: $68 \%$; $\mathrm{Mp}=116-118^{\circ} \mathrm{C} ; \mathrm{Rf}=0.75 ;{ }^{1} \mathrm{H}-\mathrm{NMR}(300 \mathrm{MHz}$, ppm, DMSO- $\left.d_{6}\right): 2.11\left(\mathrm{~s}, 3 \mathrm{H}, \mathrm{CH}_{3}\right) 2.41\left(\mathrm{~s}, 3 \mathrm{H}, \mathrm{CH}_{3}\right) 7.41(\mathrm{~d}$, $J=7.16 \mathrm{~Hz}, 1 \mathrm{H}$, arom.) 7.47-7.59 (m, 2H, arom.) 7.62-7.75 (m, 2H, arom.) 7.80-7.93 (m, 1H, arom.) 8.12 (dd, J=7.91, $1.13 \mathrm{~Hz}, 1 \mathrm{H}$, arom.); ${ }^{13} \mathrm{C}-\mathrm{NMR}$ (DMSO-d $\left.d_{6}\right) \delta$ (ppm): 161.0, $154.4,147.7,141.9,135.4,134.0,130.6,130.4,127.3$, 127.2, 126.9, 122.2, 120.7, 100.0, 23.9, 20.9; MS: $m$ / z: $329.19(\mathrm{M}+)(330.90)$.

\section{3-(3-CHLOROPHENYL)-2-METHYLQUINAZOLIN- 4(3H)-ONE (5)}

Yield: $51 \%$; $\mathrm{Mp}=113-115{ }^{\circ} \mathrm{C}$; ${ }^{1} \mathrm{H}-\mathrm{NMR}(300 \mathrm{MHz}, \mathrm{ppm}$, DMSO- $\left.d_{6}\right): 2.15\left(\mathrm{~s}, 3 \mathrm{H}, \mathrm{CH}_{3}\right), 7.42-7.73(\mathrm{~m}, 6 \mathrm{H}$, arom.), 7.79-7.90 (m, 1H, arom.), 8.10 (d, J=7.53 Hz, $1 \mathrm{H}$, arom.); ${ }^{13} \mathrm{C}-\mathrm{NMR}$ (DMSO- $d_{6}$ ) $\delta$ (ppm): 161.8, 154.4, 147.8,
139.7, 135.2, 134.1, 131.6, 129.6, 129.2, 128.0, 127.2, 126.9, 126.8, 120.9, 120.4, 24.5; MS: $m$ / z: $270.90(\mathrm{M}+)$ (270.71).

\section{2-(2-METHYL-4-OXOQUINAZOLIN-3(4H)-YL)- BENZOIC ACID (6)}

Yield: $49 \%$; Mp $=244-247^{\circ} \mathrm{C} ; \mathrm{Rf}=0.41 ;{ }^{1} \mathrm{H}-\mathrm{NMR}(600 \mathrm{MHz}$, ppm, DMSO-d $\left.d_{6}\right) 2.11(\mathrm{~s}, 3 \mathrm{H}), 7.51(\mathrm{t}, J=6.97 \mathrm{~Hz}, 1 \mathrm{H}$, arom.), 7.57 (d, $J=8.07 \mathrm{~Hz}, 1 \mathrm{H}$, arom.), 7.65-7.70 (m, 2H, arom.), 7.78-7.87 (m, 2H, arom.), 8.06-8.14 (m, $1 \mathrm{H}$, arom.); ${ }^{13} \mathrm{C}-$ NMR (DMSO- $d_{6}$ ) $\delta$ (ppm): 165.7, 161.3, 154.2, 147.5, 137.5, $134.5,133.6,131.5,130.2,129.5,128.9,126.6,126.2$, 120.4, 23.7; MS: $281.00 \mathrm{~m} / \mathrm{z}$ : $(\mathrm{M}+)(280.28)$.

\section{2-METHYL-3-PHENYLQUINAZOLIN-4(3H)-ONE (7)} Yield: $74 \%$; $\mathrm{Mp}=127-128{ }^{\circ} \mathrm{C} ; \mathrm{Rf}=0.62 ;{ }^{1} \mathrm{H}-\mathrm{NMR}(600 \mathrm{MHz}$, ppm, DMSO- $\left.d_{6}\right): 2.13\left(\mathrm{~s}, 3 \mathrm{H}, \mathrm{CH}_{3}\right), 7.43-7.47(\mathrm{~m}, 2 \mathrm{H}$, arom.), 7.49-7.54 (m, 2H, arom.), 7.55-7.60 (m, 2H, arom.), 7.67 (d, $J=8.07 \mathrm{~Hz}, 1 \mathrm{H}$, arom.), 7.82-7.86 (m, $1 \mathrm{H}$, arom.), 8.10 (dd, $J=8.07,1.47 \mathrm{~Hz}, 1 \mathrm{H}$, arom.); ${ }^{13} \mathrm{C}-\mathrm{NMR}$ (DMSO- $\left.d_{6}\right) \delta$ (ppm): $161.3,154.4,147.3,137.9,136.5,129.5,128.9,128.4$ 126.6, 126.4, 126.3, 120.5, 24.0; MS: $m$ / z: $237.00(\mathrm{M}+)$ (236.27).

\section{3-(4-METHOXYPHENYL)-2-METHYL-6-NITROQUINAZOLIN- 4(3H)-ONE (8)}

Yield: $84 \% ; \mathrm{Mp}=229-230{ }^{\circ} \mathrm{C} ; \mathrm{Rf}=0.70 ;{ }^{1} \mathrm{H}-\mathrm{NMR}(300 \mathrm{MHz}$, ppm, DMSO- $\left.d_{6}\right): 2.20\left(\mathrm{~s}, 3 \mathrm{H}, \mathrm{CH}_{3}\right), 3.85\left(\mathrm{~s}, 3 \mathrm{H}, \mathrm{OCH}_{3}\right), 7.11-$ $7.14(\mathrm{~d}, 2 \mathrm{H}, J=9.04$, arom.), 7.40-7.43 (d, $2 \mathrm{H}, J=8.67$, arom.), $7.83(\mathrm{~d}, 1 \mathrm{H}, J=9.04$, arom.), 8.55 (dd, $1 \mathrm{H}, J=9.04$, 2.64, arom.), $8.76\left(\mathrm{~s}, 1 \mathrm{H}\right.$, arom.); ${ }^{13} \mathrm{C}-\mathrm{NMR}$ (DMSO- $d_{6}$ ) $\delta$ (ppm): 161.3, 160.0, 159.7, 152.0, 145.1, 130.2, 129.9, 128.9, 122.9, 121.1, 115.3, 55.9, 24.9; MS: $m$ / z: 312.20 $(\mathrm{M}+)(311.29)$.

\section{6-IODO-3-(4-METHOXYPHENYL)-2-METHYLQUINAZOLIN- 4(3H)-ONE (9)}

Yield: $63 \%$; $\mathrm{Mp}=141-143^{\circ} \mathrm{C} ; \mathrm{Rf}=0.69 ;{ }^{1} \mathrm{H}-\mathrm{NMR}(300 \mathrm{MHz}$, ppm, DMSO- $\left.d_{6}\right): 2.12\left(\mathrm{~s}, 3 \mathrm{H}, \mathrm{CH}_{3}\right), 3.83\left(\mathrm{~s}, 3 \mathrm{H}, \mathrm{OCH}_{3}\right), 7.04-$ 7.15 (m, 2H, arom.), 7.28-7.38 (m, 2H, arom.), 7.44 (d, $J=8.67 \mathrm{~Hz}, 1 \mathrm{H}$, arom.), 8.11 (dd, $J=8.48,2.07 \mathrm{~Hz}, 1 \mathrm{H}$, arom.), 8.35 (d, J=2.26 Hz, $1 \mathrm{H}$, arom.); ${ }^{13} \mathrm{C}-\mathrm{NMR}$ (DMSO- $d_{6}$ ) $\delta$ (ppm): $160.7,159.8,156.3,147.1,143.3,135.0,130.6,129.9$, 129.3, 122.9, 115.2, 115.1, 91.5, 55.6, 24.7; MS: $m$ / z: $393.00(\mathrm{M}+)(392.19)$

\section{2-((4-METHYL-2-OXO-2H-CHROMEN-7-YL)OXY)-N-(2-} METHYL-4-OXOQUINAZOLIN-3(4H)-YL)ACETAMIDE (10) Yield: $75 \%$; $\mathrm{Mp}=230{ }^{\circ} \mathrm{C} ; \mathrm{Rf}=0.28 ;{ }^{1} \mathrm{H}-\mathrm{NMR}(600 \mathrm{MHz}, \mathrm{ppm}$, DMSO- $\left.d_{6}\right): 2.11\left(\mathrm{~s}, 3 \mathrm{H}, \mathrm{CH}_{3}\right), 2.36\left(\mathrm{~s}, 3 \mathrm{H}, \mathrm{CH}_{3}\right), 5.01(\mathrm{~s}, 2 \mathrm{H}$, $\mathrm{CH}_{2}$ ), 6.20 (s, $1 \mathrm{H}$; C-3 coum.), 6.83 (dd, J=8.80, $2.2 \mathrm{~Hz}, 1 \mathrm{H}$, arom.), 6.93 (s, $1 \mathrm{H}$, arom.), 7.54 (d, J= $8.07 \mathrm{~Hz}, 1 \mathrm{H}$, arom.), $7.61(\mathrm{~d}, J=8.80 \mathrm{~Hz}, 1 \mathrm{H}$, arom.), $7.66(\mathrm{~m}, 1 \mathrm{H}$, arom.), 7.76 
(m, 1H, arom.), 8.05 (m, $1 \mathrm{H}$, arom.); ${ }^{13} \mathrm{C}-\mathrm{NMR}$ (DMSO- $\left.d_{6}\right) \delta$ (ppm): 165.5, 160.2, 159.9, 154.3, 153.2, 152.8, 149.4, 133.3, 132.4, 131.4, 130.4, 129.6, 129.4, 126.3, 113.6, 112.2, 111.44, 101.6, 60.5, 18.1, 10.4; MS: $m$ / z: 392.20 $(\mathrm{M}+)(391.38)$.

\section{2-(7-HYDROXY-2-OXO-2H-CHROMEN-4-YL)-N-(2-METHYL- 4-OXOQUINAZOLIN-3(4H)-YL)ACETAMIDE (11)}

Yield: $79 \% ; \mathrm{Mp}=200-204{ }^{\circ} \mathrm{C} ; \mathrm{Rf}=0.30 ;{ }^{1} \mathrm{H}-\mathrm{NMR}(300 \mathrm{MHz}$, ppm, DMSO- $\left.d_{6}\right): 2.36\left(\mathrm{~s}, 3 \mathrm{H}, \mathrm{CH}_{3}\right), 4.02\left(\mathrm{~s}, 2 \mathrm{H},-\mathrm{CH}_{2}-\right), 6.39$ (s, $1 \mathrm{H}$, coum.), $6.75(\mathrm{~s}, 1 \mathrm{H}$, arom.), $6.83(\mathrm{~m}, 1 \mathrm{H}$, arom.), 7.53 (m, $1 \mathrm{H}$, arom.), 7.63 (d, $1 \mathrm{H}, J=7.91$, arom.), 7.73 (d, $1 \mathrm{H}, J=$ 8.67, arom.), 7.85 (m, $1 \mathrm{H}$, arom.), 8.10 (dd, $1 \mathrm{H}, J=7.91$ 1.13, arom); ${ }^{13} \mathrm{C}-\mathrm{NMR}$ (DMSO- $d_{6}$ ) $\delta$ (ppm): 168.4, 161.8, $160.6,159.3,156.3,155.5,150.1,147.0,135.5,120.9$, 113.4, 112.8, 111.7, 102.9, 37.1, 21.5; MS: $m$ / z: 376.20 (M-) (377.35).

\section{N,N'-BIS(2-METHYL-4-OXOQUINAZOLIN-3(4H)- YL)PYRIDINE-2,6-DICARBOXAMIDE (12)}

Yield: $31 \%$; $M p=225-228^{\circ} \mathrm{C} ; \mathrm{Rf}=0.20 ;{ }^{1} \mathrm{H}-\mathrm{NMR}(300 \mathrm{MHz}$, ppm, DMSO-d6): $2.53\left(\mathrm{~s}, 3 \mathrm{H}, \mathrm{CH}_{3}\right), 2.55\left(\mathrm{~s}, 3 \mathrm{H}, \mathrm{CH}_{3}\right), 7.59$ (t, $J=7.54 \mathrm{~Hz}, 2 \mathrm{H}$, aom.), 7.73 (d, J=8.29 Hz, $2 \mathrm{H}$, arom.), 7.91 (t, $J=7.54 \mathrm{~Hz}, 2 \mathrm{H}$, arom.), 8.10-8.23 (m, $2 \mathrm{H}$, arom.), 8.36-8.50 (m, 3H, arom.); ${ }^{13} \mathrm{C}-\mathrm{NMR}$ (DMSO- $\left.d_{6}\right) \delta$ (ppm): 162.9, 162.8, $159.5,156.5,147.2,147.1,147.0,141.2,135.8,127.6$, 127.0, 120.8, 21.7; MS: $m$ / z: 482.10 (M+) (481.46).

\section{3-(4-(TRIFLUOROMETHOXY)PHENYL)QUINAZOLIN- 4(3H)-ONE (13)}

Yield: $48 \%$; $\mathrm{Mp}=138-139{ }^{\circ} \mathrm{C} ; \mathrm{Rf}=0.76 ;{ }^{1} \mathrm{H}-\mathrm{NMR}(600 \mathrm{MHz}$, ppm, DMSO- $\left.d_{6}\right)$ : 7.56-7.60 (m, 3H, arom.), 7.70-7.74 $(\mathrm{m}, 3 \mathrm{H}$, arom.), 7.86-7.89 (m, $1 \mathrm{H}$, arom.), $8.19(\mathrm{~m}, 1 \mathrm{H}$, arom.), 8.36 (s, $1 \mathrm{H}, \mathrm{C}-2) ;{ }^{13} \mathrm{C}-\mathrm{NMR}$ (DMSO- $\left.d_{6}\right) \delta$ (ppm): $159.9,147.7,136.5,134.7,129.7,127.5,127.3,126.4$, 121.8;MS: $m$ / z: $307.10(\mathrm{M}+)$ (306.24).

\section{2-(4-OXOQUINAZOLIN-3(4H)-YL)BENZOIC ACID (14) ${ }^{[36]}$} Yield: $35 \%$; $M p=295-297^{\circ} \mathrm{C}$ (lit. $287-288^{\circ} \mathrm{C}$ ); Rf $=0.48 ;{ }^{1} \mathrm{H}$ NMR (600 MHz, ppm, DMSO- $\left.d_{6}\right): 7.68-7.72(\mathrm{~m}, 2 \mathrm{H}$, arom.), $7.77(\mathrm{~m}, 1 \mathrm{H}$, arom.), $7.84(\mathrm{~m}, 1 \mathrm{H}$, arom.), 7.88-7.92 (m, 1H, arom.), 7.98 (m, $1 \mathrm{H}$, arom.), 8.18 (dd, $J=8.07,1.4 \mathrm{~Hz}, 1 \mathrm{H}$, arom.), 8.27 (dd, $J=8.07,1.4 \mathrm{~Hz}, 1 \mathrm{H}$, arom.), $8.42(\mathrm{~s}, 1 \mathrm{H}, \mathrm{C}$ 2), 13.18 (br.s., $1 \mathrm{H}, \mathrm{OH}$ ); ${ }^{13} \mathrm{C}-\mathrm{NMR}$ (DMSO-d $\left.d_{6}\right) \delta$ (ppm): $165.8,160.3,147.9,147.1,137.1,134.6,133.4,131.1$, 129.5, 129.2, 127.2, 126.3, 121.9; MS: $m$ / z: $267.00(\mathrm{M}+)$ (266.25).

\section{3-(2,5-DIMETHOXYPHENYL)QUINAZOLIN-4(3H)-ONE (15)} Yield: $54 \% ; \mathrm{Mp}=159-161{ }^{\circ} \mathrm{C} ; \mathrm{Rf}=0.71 ;{ }^{1} \mathrm{H}-\mathrm{NMR}(300 \mathrm{MHz}$, ppm, DMSO- $\left.d_{6}\right): 3.72\left(\mathrm{~s}, 3 \mathrm{H}, \mathrm{OCH}_{3}\right), 3.76\left(\mathrm{~s}, 3 \mathrm{H}, \mathrm{OCH}_{3}\right), 7.11$ (m, $1 \mathrm{H}$, arom.), 7.15-7.21 (m,2H, arom.), 7.60 (t, $J=6.97$ $\mathrm{Hz}, 1 \mathrm{H}$, arom.), 7.76 (d, $J=8.29 \mathrm{~Hz}, 1 \mathrm{H}$, arom.), $7.89(\mathrm{~m}, 1 \mathrm{H}$,
$=\mathrm{CH}), 8.20(\mathrm{~s}, 1 \mathrm{H}, \mathrm{C}-2) ;{ }^{13} \mathrm{C}-\mathrm{NMR}\left(\mathrm{DMSO}-d_{6}\right) \delta(\mathrm{ppm}): 160.2$, $149.1,148.4,135.1,127.8,126.8,122.5,116.0,115.8$, 113.9, 56.7, 56.2; MS: $m$ / z: $282.90(\mathrm{M}+)$ (282.29).

\section{3-PHENYLQUINAZOLIN-4(3H)-ONE (16) ${ }^{[37]}$}

Yield: $12 \%$; $\mathrm{Mp}=143-145^{\circ} \mathrm{C}$ (lit. $139^{\circ} \mathrm{C}$ ); Rf $=0.73 ;{ }^{1} \mathrm{H}-\mathrm{NMR}$ (300 MHz, ppm, DMSO- $d_{6}$ ): 7.55-7.63 (m, 6H, arom.), 7.75 ( $\mathrm{m}, 1 \mathrm{H}$, arom.), 7.86 (dd, $J=6.97,1.3 \mathrm{~Hz}, 1 \mathrm{H}$, arom.), 8.21 (dd, $J=8.10,0.9 \mathrm{~Hz}, 1 \mathrm{H}$, arom.), 8.36 (s, $1 \mathrm{H}, \mathrm{C}-2) . ;{ }^{13} \mathrm{C}-\mathrm{NMR}$ (DMSO-d $\left.d_{6}\right) \delta$ (ppm): 160.44, 148.2, 147., 138.1, 135.1, 129.7, 129.2, 127.9, 127.8, 127.7, 126.9, 122.43; MS: $m$ / z: $221.10(\mathrm{M}-)(222.24)$.

\section{3-(3-CHLOROPHENYL)QUINAZOLIN-4(3H)-ONE (17)} Yield: $57 \%$; $\mathrm{Mp}=155-157{ }^{\circ} \mathrm{C} ; \mathrm{Rf}=0.731 \mathrm{NMR}(600 \mathrm{MHz}$, ppm, DMSO-d6): 7.54-7.57 (m, 1H, arom.), 7.59-7.62 (m, $3 \mathrm{H}$, arom.), 7.74-7.77 (m, 2H, arom.), 7.87-7.92 (m, $1 \mathrm{H}$, arom.), 8.21 (dd, J=8.07, $1.47 \mathrm{~Hz}, 1 \mathrm{H}$, arom.), 8.37 (s, $1 \mathrm{H}, \mathrm{C}$ 2); ${ }^{13} \mathrm{C}-\mathrm{NMR}\left(\mathrm{DMSO}-d_{6}\right) \delta$ (ppm): 159.9, 149.7, 146.8, 138.9, $134.7,134.6,133.2,130.8,128.8,127.8,127.5,127.3$, 126.4, 123.3, 121.8, 118.7; MS: $m$ / z: $257.39(\mathrm{M}+)$ (256.69).

\section{RESULTS AND DISCUSSION}

Deep eutectic solvents have found a great application in organic synthesis. Among many, choline chloride based deep eutectic solvents have proven to be very effective in various organic transformations. When choline chloride is combined with various HBDs, a great number of DESs is formed, all differing in their chemical and physical properties. Therefore, in order to find the most suitable DES for desired chemical reaction, the best way is to perform a screening of different DESs on a model reaction.

In order to find the most efficient reaction media for green synthesis of different quinazolinones, the model reaction of benzoxazinone and 4-chloroaniline (Figure 2) was performed, in twenty choline chloride based deep eutectic solvents (Table 2.). It was found that DESs are not suitable for the synthesis of the intermediate benzoxazionone,

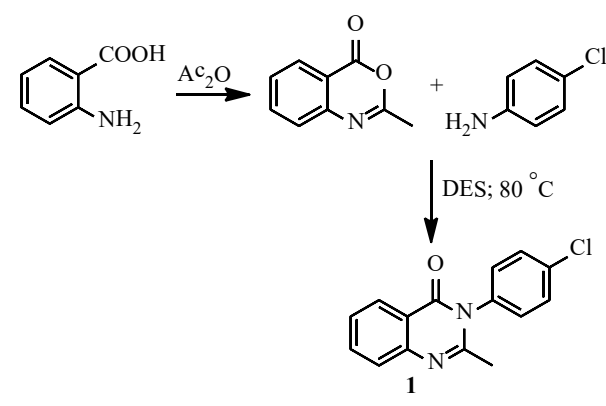

Figure 2. Model reaction for the synthesis of benzoxazinone. 
because only $\mathrm{N}$-acetylanthranilic acid was obtained. Therefore, benzoxazinone was prepared conventionally, ${ }^{[38]}$ via condensation reaction of anthranilic acid and acetic anhydride under reflux for 4 hours. The product was obtained after evaporation of the excess of acetic anhydride and acetic acid, washed with petroleum ether and immediately used for all further reactions. The reaction optimization was performed with 4-chloroaniline in DESs in the presence of molecular sieves during $2-4$ hours at $80{ }^{\circ} \mathrm{C}$. The results are tabulated in Table 1.

${ }^{1} \mathrm{H}$ and ${ }^{13} \mathrm{C}$ NMR spectra elucidated structures of all the synthesized compounds. Methyl protons of all 2methylquinazolinone derivatives show characteristic singlet peak at 2.11-2.36 ppm. Aromatic protons are visible at 7.01-8.76 ppm. All characteristic peaks for phenyl ring substituents are also present; methoxy protons for compound $\mathbf{2}$ at $3.85 \mathrm{ppm}$, methyl protons for compound $\mathbf{4}$ at $2.41 \mathrm{ppm}$, methoxy protons for compound 8 at $3.85 \mathrm{ppm}$ and for compound $\mathbf{9}$ at $3.83 \mathrm{ppm}$. Phenyl ring substituents influenced the product yields, where the highest yield was obtained with 4-chlorophenyl aniline, and the lowest in

Table 1. Choline chloride based deep eutectic solvents with different HBDs in green synthesis of 3-(4-chlorophenyl)-2methylquinazolinone (1).

\begin{tabular}{|c|c|c|c|c|}
\hline Entry & $\mathrm{HBD}$ & $\begin{array}{c}\text { Ratio } \\
\text { HBD : HBA }\end{array}$ & Ref. & Yield / \% \\
\hline 1 & Urea & $1: 2$ & 18 & 89 \\
\hline 2 & $N$-methylurea & $1: 3$ & 18 & 73 \\
\hline 3 & $\begin{array}{c}\text { 1,3- } \\
\text { dimethylurea }\end{array}$ & $1: 2$ & 39 & 87 \\
\hline 4 & Thiourea & $1: 2$ & 18 & 64 \\
\hline 5 & Acetamide & $1: 2$ & 18 & 62 \\
\hline 6 & Butane-1,4-diol & $1: 3$ & 40 & 57 \\
\hline 7 & Ethane-1,2-diol & $1: 2$ & 18 & 42 \\
\hline 8 & Glycerol & $1: 2$ & 41 & 36 \\
\hline 9 & Xylitol & $1: 1$ & 39 & 44 \\
\hline 10 & Sorbitol & $1: 1$ & 41 & 64 \\
\hline 11 & Glucose & $2: 1$ & 41 & in traces \\
\hline 12 & Fructose & $2: 1$ & 41 & in traces \\
\hline 13 & Citric acid & $1: 1$ & 39 & in traces \\
\hline 14 & Tartaric acid & $1: 1$ & 42 & 55 \\
\hline 15 & Oxalic acid & $1: 1$ & 41 & 62 \\
\hline 16 & Levulinic acid & $1: 2$ & 39 & in traces \\
\hline 17 & Lactic acid & $1: 2$ & 43 & 50 \\
\hline 18 & Malic acid & $1: 1$ & 41 & 71 \\
\hline 19 & Malonic acid & $1: 1$ & 41 & 79 \\
\hline 20 & Maleic acid & $1: 1$ & 44 & 61 \\
\hline
\end{tabular}

compound 12, with dipicolinic acid hydrazide as a starting compound. Compounds $\mathbf{1 0}$ and $\mathbf{1 1}$ were successfully synthesized, although the starting amino compounds were bulky molecules, thus forming a quinazolinone-coumarin hybrids. For compound $\mathbf{1 0}$ coumarin proton of $\mathrm{C}-3$ peaked at $6.20 \mathrm{ppm}$, while two $-\mathrm{CH}_{2^{-}}$protons are found at $5.01 \mathrm{ppm}$. For compound $\mathbf{1 1}$ coumarin $\mathrm{C}-3$ proton is found at $6.39 \mathrm{ppm}$, while two $-\mathrm{CH}_{2}$-protons are found at $4.02 \mathrm{ppm}$.

Second part of this research involved the synthesis of 3-aryl quinazolinones (13-17), starting from anthranilic acid, trimethyl orthoformate and different aromatic amines (Figure 4).

These reactions demanded higher temperatures, $120{ }^{\circ} \mathrm{C}$, respectively, which cannot be accomplished in DESs, due to their degradation at higher temperatures. Therefore, DES could not be efficiently employed for their synthesis. Here we decided to apply microwaves, in order to maintain the green character of the process. So far, there are several examples of 3-aryl quinazolinone synthesis from

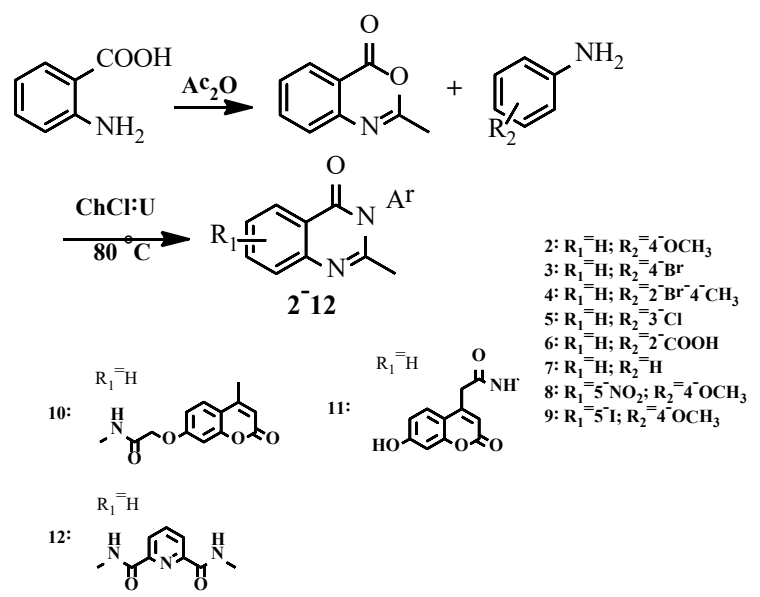

Figure 3. Synthesis of 2-methyl-3-substituted- quinazolinones.

Table 2. Reaction time and yield of synthesized compounds.

\begin{tabular}{cccccc}
\hline Comp. & Time / min & Yield /\% & Comp. & Time / min & Yield /\% \\
\hline 1 & 120 & 89 & 10 & 240 & 75 \\
2 & 150 & 71 & 11 & 240 & 79 \\
3 & 150 & 53 & 12 & 240 & 31 \\
4 & 150 & 68 & 13 & 30 & 48 \\
5 & 150 & 51 & 14 & 30 & 35 \\
6 & 180 & 49 & 15 & 30 & 54 \\
7 & 180 & 74 & 16 & 30 & 12 \\
8 & 240 & 84 & 17 & 30 & 57 \\
9 & 240 & 63 & & & \\
\hline
\end{tabular}




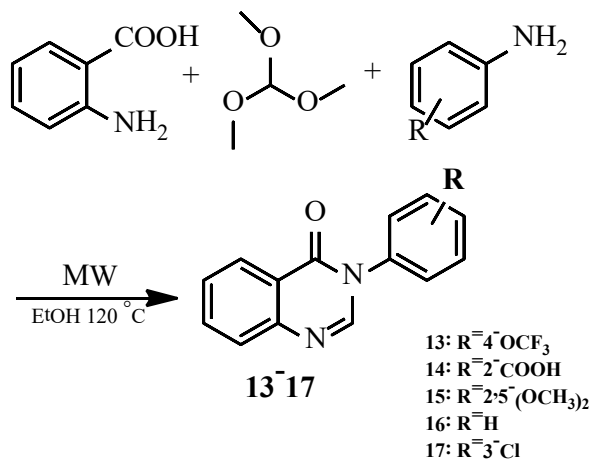

Figure 4. Microwave synthesis of 3-substituted quinazolinones.

anthranilic acid. Catalysts such as DABCO, ${ }^{[36]}$ heteropolyacids, ${ }^{[37]}$ lanthanum(III) nitrate hexahydrate or $p$-toluenesulfonic acid ${ }^{[45]}$ are commonly used, as well as different organic solvents, like DMF with sulfuric acid under reflux ${ }^{[46]}$ or phosphoryl chloride in DMF.[47] Inspired by these methods, our intention was to synthesize 3-aryl quinazolinones without any catalyst or toxic solvents, using microwaves. All reactions were carried out in ethanol at $120{ }^{\circ} \mathrm{C}$ for 30 minutes. ${ }^{1} \mathrm{H}$ NMR spectra shows a characterristic peaks for the synthesized compounds. Quinazolinone C-2 proton shows a singlet at $8.20-8.42 \mathrm{ppm}$. Aromatic peaks are visible in a range of 7.11-8.27 ppm for all compounds. Different substituents for the compounds also exhibit a characteristic peaks; carboxylic $-\mathrm{OH}$ group for compound 14 at $13.18 \mathrm{ppm}$, methoxy groups for compound 15 at 3.72 and $3.76 \mathrm{ppm}$.

All of the synthesized compounds are compared to the ones known in the literature, and their structure data correspond to the literature ones, as well as the melting points.

\section{CONCLUSIONS}

We have described two efficient green approaches for the synthesis of 3-aryl and 2-methyl-3-substituted quinazolinones, from anthranilic acid, in mild conditions. A good selection of solvent and method can lead to the faster and more successful synthesis. The present methods show many advantages compared to the previously reported in literature. Short reaction time, no use of catalyst, simplicity of product isolation and environmental aspect are the advantages of both methods applied when compared to the literature methods.

Acknowledgment. This work has been supported in part by Croatian Science Foundation under the project "Green Technologies in Synthesis of Heterocyclic Compounds" (UIP2017-05-6593).

\section{REFERENCES}

[1] S. K. Sahu, Md. Afzal Azam, M. Banerjee, S. Acharrya, C. C. Behera, S. Si, J. Braz. Chem. Soc. 2008, 19, 963970.

https://doi.org/10.1590/\$0103-50532008000500023

[2] A. N. Babu, R. R. Nadendla, J. Pharm. Res. 2011, 4, 983-985.

[3] P. Mishra, P. N. Gupta, A. K. Shakya, J. Ind. Chem. Soc. 1991, 68, 618-619.

[4] M. A. Sayyed, S. S. Mokle, Y. B. Vibhute, Arkivoc 2006, 11, 221-226.

[5] H. Y. Hassan, A. A. Ismaiel, H. A. H. El-Sherief, Eur. J. Med. Chem. 1991, 26, 743-748.

https://doi.org/10.1016/0223-5234(91)90126-8

[6] P. Panneerselvam, R. V. Pradeepchandran, S. K. Sridhar, Indian. J. Pharm. Sci. 2003, 65, 268-273.

[7] L. Hosseinzadeh, A. Aliabadi, M. Kalantri, A. Mostafavi, M. R. Khajouei, Res. Pharm. Sci. 2016, 11, 210.

[8] S. Poorirani, S. Sadeghian-Rizi, G. Khodarahmi, M. Rahmani Khajouei, F. Hassanzadeh, Res. Pharm. Sci. 2018, 13, 450-459.

https://doi.org/10.4103/1735-5362.236838

[9] F. M. Refaie, A. Y. Esmat, S. M. Abdel Gawad, A. M. Ibrahim, M. A. Mohamed, Lipids in Health and Disease 2005, 4, 22-30.

https://doi.org/10.1186/1476-511X-4-22

[10] H. D. Revanasiddappa, K. S. Prasad, L. S. Kumar, B. Jayalakshmi, Int. J. Chem. Tech. Res. 2010, 2, 13441349.

[11] P. T. Anastas, J. C. Warner, Green Chemistry Theory and Practice, Oxford University Press, Oxford, 2000.

[12] Z. Maugeri, P. D. de Maria, RSC Adv. 2011, 2, 421425. https://doi.org/10.1039/C1RA00630D

[13] Y. Liu, J. B. Friesen, J. B. McAlpine, D. C. Lankin, S.-N. Chen, G. F. Pauli, J. Nat. Prod. 2018, 81, 679-690. https://doi.org/10.1021/acs.jnatprod.7b00945

[14] M. Molnar, H. Brahmbhatt, V. Rastija, V. Pavić, M. Komar, M. Karnaš, J. Babić, Molecules 2018, 23, 1897. https://doi.org/10.3390/molecules23081897

[15] M. Molnar, I. Periš, M. Komar, Eur. J. Org. Chem. 2019, 15, 2688-2694.

https://doi.org/10.1002/ejoc.201900249

[16] M. Molnar, M. Komar, H. Brahmbhatt, J. Babić, S. Jokić, V. Rastija, Molecules 2017, 22, 1482.

https://doi.org/10.3390/molecules22091482

[17] M. Molnar, J. Klenkar, T. Tarnai, Synth. Commun. 2017, 47, 1040-1045.

https://doi.org/10.1080/00397911.2017.1291815

[18] A. P. Abbott, D. Boothby, G. Capper, D. L. Davies, R. K. Rasheed, J. Am. Chem. Soc. 2004, 126, 9142-9147. https://doi.org/10.1021/ja048266j 
[19] Y. Dai, J. van Spronsen, G.-J. Witkamp, R. Verpoorte, Y. H. Choi, Anal. Chim. Acta 2013, 766, 61-68. https://doi.org/10.1016/j.aca.2012.12.019

[20] J. G. Alvarez, Eur. J. Inorg. Chem. 2015, 31, 51475157. https://doi.org/10.1002/ejic.201500892

[21] D. A. Alonso, A. Baeza, R. Chinchilla, G. Guillena, I. M. Pastor, D. J. Ramón, Eur. J. Org. Chem. 2016, 4, 612632. https://doi.org/10.1002/ejoc.201501197

[22] M. Francisco, A. van den Bruinhorst, M. C. Kroon, Angew. Chem. Int. Ed. 2013, 52, 3074-3085. https://doi.org/10.1002/anie.201207548

[23] N. Azizi, S. Dezfooli, M. Khajeh, M. M. Hashemi, J. Mol. Liq. 2013, 186, 76-80. https://doi.org/10.1016/j.molliq.2013.05.011

[24] H. N. Harishkumar, K. M. Mahadevan, H. C. K. Kumar, N. D. Satyanarayan, Org. Commun. 2011, 4, 26-32.

[25] F. Keshavarzipour, H. Tavakol, J. Iran Chem. Soc. 2016, 13, 149-153. https://doi.org/10.1007/s13738-015-0722-9

[26] H. R. Lobo, B. S. Singh, G. S. Shankarling, Catal. Commun. 2012, 27, 179-183.

[27] Z.-H. Zhang, X.-N. Zhang, L.-P. Mo, Y.-X. Li, F.-P. Ma, Green Chem. 2012, 14, 1502-1506. https://doi.org/10.1039/c2gc35258c

[28] S. K. Ghosh, R. Nagarajan, RSC Adv. 2016, 6, 2737827387. https://doi.org/10.1039/C6RA00855K

[29] W. A. A. Arafa, R. Soc. Open Sci. 2019, 6, 182046. https://doi.org/10.1098/rsos.182046

[30] T. S. Patel, J. D. Bhatt, R. B. Dixit, C. J. Chudasama, B. D. Patel, B. C. Dixit, Bioorg. Med. Chem. 2019, 27, 3574-3586. https://doi.org/10.1016/j.bmc.2019.06.038

[31] C. L. Jagani, N. A. Sojitra, S. F. Vanparia, T. S. Patel, B. C. Dixit, J. Saudi Chem. Soc. 2012, 16, 363-369. https://doi.org/10.1016/j.jscs.2011.02.001

[32] M. G. R. Priya, Y. Zulykama, K. Girija, S. Murugesh, P. T. Perumal, Indian J. Chem. 2011, 50B, 98-102.

[33] M. H. Hekal, F. S. M. A. El-Azm, S. R. Atta-Allah, Syn. Commun. 2019, 40, 2630-2641. https://doi.org/10.1080/00397911.2019.1637001
[34] D. Kumar, P. S. Jadhavar, M. Nautiyal, H. Sharma, P. K. Meena, L. Adane, S. Pancholia, A. K. Chakraborti, RSC Adv. 2015, 5, 30819-30825.

https://doi.org/10.1039/C5RA03888J

[35] A. P. Abbott, G. Capper, D. L. Davies, R. K. Rasheeda, V. Tambyrajaha, Chem. Commun. 2003, 70-71. https://doi.org/10.1039/b210714g

[36] M. M. Heravi, N. Javanmardi, H. A. Oskooie, B. Baghernejad, Bull. Chem. Ethiop. 2011, 25, 305-308. https://doi.org/10.4314/bcse.v25i2.65909

[37] K. Ighilahriz, B. Boutemeur, F. Chami, C. Rabia, M. Hamdi, Molecules 2008, 13, 779-789. https://doi.org/10.3390/molecules13040779

[38] T. Singh, S. Sharma, V. K. Srivastava, A. Kumar, Indian J. Chem. B: Org. Med. Chem. 2006, 45, 2558-2565.

[39] Q. Zhang, K. D. O. Vigier, S. Royer, F. Jerome, Chem. Soc. Rev. 2012, 41, 7108-7146. https://doi.org/10.1039/c2cs35178a

[40] P. Liu, J.-W. Hao, L.-P. Mo, Z.-H. Zhang, RSC Adv. 2015, 5, 48675-48704. https://doi.org/10.1039/C5RA05746A

[41] F. S. Mjalli, J. Taiw. Inst. Chem. Eng. 2016, 61, 64-74. https://doi.org/10.1016/j.jtice.2015.12.020

[42] G. Garcia, S. Aparicio, R. Ullah, M. Atilhan, Energy Fuels 2015, 29, 2616-2644. https://doi.org/10.1021/ef5028873

[43] A. Skulcova, V. Majova, A. Haz, F. Kreps, A. Russ, M. Jablonsky, Int. J. Sci. Eng. Res. 2017, 8, 2249-2252.

[44] H. Malaeke, M. R. Housaindokht, H. Monhemi, M. Izadyar, J. Mol. Liq. 2018, 263, 193-199. https://doi.org/10.1016/j.molliq.2018.05.001

[45] M. Narashimulu, K. C. Mahesh, T. S. Reddy, K. Rajesh, Y. Venkateswarlu, Tetrahedron Lett. 2006, 47, 43814383. https://doi.org/10.1016/j.tetlet.2006.04.096

[46] S.-L. Cao, M. Zhang, Y.-P. Feng, Y.-Y. Jiang, N. Zhang, Syn. Commun. 2008, 38, 2227-2236. https://doi.org/10.1080/00397910802026584

[47] V. J. Majo, P. T. Perumal, Tetrahedron Lett. 1996, 37, 5015-5018.

https://doi.org/10.1016/0040-4039(96)01022-2 